\title{
Identification of the crossing point at $N=21$ between normal and intruder configurations
}

R. Lică, ${ }^{1,2}$ F. Rotaru, ${ }^{2}$ M. J. G. Borge, ${ }^{1,3}$ S. Grévy, ${ }^{4,5}$ F. Negoiţă ${ }^{2}$ A. Poves, ${ }^{6,7}$ O. Sorlin, ${ }^{4}$ A. N. Andreyev, ${ }^{8}$ R. Borcea, ${ }^{2}$

C. Costache,${ }^{2}$ H. De Witte, ${ }^{9}$ L. M. Fraile, ${ }^{10}$ P. T. Greenlees,,${ }^{11,12}$ M. Huyse, ${ }^{9}$ A. Ionescu, ${ }^{2}$ S. Kisyov, ${ }^{2,13}$ J. Konki, ${ }^{11,12}$ I. Lazarus, ${ }^{14}$ M. Madurga, ${ }^{1}$ N. Mărginean, ${ }^{2}$ R. Mărginean, ${ }^{2}$ C. Mihai, ${ }^{2}$ R. E. Mihai, ${ }^{2}$ A. Negret, ${ }^{2}$ R. D. Page, ${ }^{15}$ J. Pakarinen,,${ }^{11,12}$ S. Pascu, ${ }^{2}$ V. Pucknell, ${ }^{14}$ P. Rahkila, ${ }^{11,12}$ E. Rapisarda, ${ }^{1}$ A. Şerban, ${ }^{2}$ C. O. Sotty, ${ }^{2}$ L. Stan, ${ }^{2}$ M. Stănoiu, ${ }^{2}$ O. Tengblad, ${ }^{3}$ A. Turturică, ${ }^{2}$ P. Van Duppen, ${ }^{9}$ R. Wadsworth, ${ }^{8}$ and N. Warr ${ }^{16}$

(IDS Collaboration)

${ }^{1}$ CERN, CH-1211 Geneva 23, Switzerland

2 “Horia Hulubei” National Institute for Physics and Nuclear Engineering, RO-077125 Bucharest, Romania

${ }^{3}$ Instituto de Estructura de la Materia, CSIC, Serrano 113 bis, E-28006 Madrid, Spain

${ }^{4}$ GANIL, CEA/DRF-CNRS/IN2P3, Bvd Henri Becquerel, 14076 Caen, France

${ }^{5}$ UMR 5797, CNRS/IN2P3, Université de Bordeaux, Chemin du Solarium, 33175 Gradignan Cedex, France

${ }^{6}$ Departamento de Física Teórica, Universidad Autónoma de Madrid, E-28049 Madrid, Spain

${ }^{7}$ Instituto de Física Teórica, UAM-CSIC E-28049 Madrid, Spain

${ }^{8}$ Department of Physics, University of York, York YO10 5DD, $N$ Yorkshire, United Kingdom

${ }^{9}$ KU Leuven, Instituut voor Kern- en Stralingsfysica, Celestijnenlaan 200D, 3001 Leuven, Belgium

${ }^{10}$ Grupo de Física Nuclear, Facultad de CC. Físicas, Universidad Complutense, CEI Moncloa, 28040 Madrid, Spain

${ }^{11}$ Department of Physics, University of Jyvaskyla, P.O. Box 35, FI-40014 University of Jyvaskyla, Finland

${ }^{12}$ Helsinki Institute of Physics, University of Helsinki, P.O. Box 64, FIN-00014 Helsinki, Finland

${ }^{13}$ Faculty of Physics, University of Sofia "St. Kliment Ohridski," 1164 Sofia, Bulgaria

${ }^{14}$ STFC Daresbury, Daresbury, Warrington WA4 4AD, United Kingdom

${ }^{15}$ Department of Physics, Oliver Lodge Laboratory, University of Liverpool, Liverpool L69 7ZE, United Kingdom

${ }^{16}$ Institut für Kernphysik, Universität zu Köln, Zülpicher Strasse 77, D-50937 Köln, Germany

(Received 13 December 2016; published 9 February 2017)

\begin{abstract}
The $\beta^{-}$decay of ${ }^{34} \mathrm{Mg}$ was used to study the ${ }^{34} \mathrm{Al}$ nucleus through $\gamma$ spectroscopy at the Isotope Separator OnLine facility of CERN. Previous studies identified two $\beta$-decaying states in ${ }^{34} \mathrm{Al}$ having spin-parity assignments $J^{\pi}=4^{-}$dominated by the normal configuration $\pi\left(d_{5 / 2}\right)^{-1} \otimes v\left(f_{7 / 2}\right)$ and $J^{\pi}=1^{+}$by the intruder configuration $\pi\left(d_{5 / 2}\right)^{-1} \otimes v\left(d_{3 / 2}\right)^{-1}\left(f_{7 / 2}\right)^{2}$. Their unknown ordering and relative energy have been the subject of debate for the placement of ${ }^{34} \mathrm{Al}$ inside or outside the $N=20$ "island of inversion." We report here that the $1^{+}$intruder lies only $46.6 \mathrm{keV}$ above the $4^{-}$ground state. In addition, a new half-life of $T_{1 / 2}=44.9(4) \mathrm{ms}$, that is twice as long as the previously measured $20(10) \mathrm{ms}$, has been determined for ${ }^{34} \mathrm{Mg}$. Large-scale shell-model calculations with the recently developed SDPF-U-MIX interaction are compared with the new data and used to interpret the mechanisms at play at the very border of the $N=20$ island of inversion.
\end{abstract}

DOI: 10.1103/PhysRevC.95.021301

The existence of special numbers of constituents (called magic numbers) that give rise to enhanced stability in atoms and atomic nuclei can be explained using very simplified meanfield descriptions [1,2]. This magicity gives rise to remarkable properties in atomic nuclei, such as having a spherical shape, an enhanced $2_{1}^{+}$state energy, a reduced transition probability $B\left(E 2: 0^{+} \rightarrow 2_{1}^{+}\right)$, and displaying drops in neutron or/and proton separation energy values [3-5]. However, much more complex interactions are required to describe changes in magic numbers in nuclei having a large proton-to-neutron imbalance [6].

In the shell-model framework, the spherical mean field of an atomic nucleus is governed primarily by the monopole

Published by the American Physical Society under the terms of the Creative Commons Attribution 4.0 International license. Further distribution of this work must maintain attribution to the author(s) and the published article's title, journal citation, and DOI. part of the Hamiltonian. This term can be decomposed into central, spin-orbit, and tensor components [7,8], each of which modifies the amplitude of spherical shell gaps. The rest of the nucleon-nucleon interaction is contained in the multipole terms of the Hamiltonian dominated by the pairing and the quadrupole-quadrupole interactions [9]. The latter acts more efficiently between nucleons located in orbits whose angular momenta differ by two units of $\hbar$ [10]. It plays a decisive role in the onset of deformation and in the disappearance of magicity far from stability, in particular, when the spherical shell gap is reduced by monopole-driven effects. In this description, the spherical closed-shell configuration has a zero particle-hole character $(0 \mathrm{p}-0 \mathrm{~h})$, whereas the deformed configuration has multiparticle-multihole $n \mathrm{p}-n \mathrm{~h}$ excitations from the normally occupied to the valence orbits. Nuclei near the magic neutron number $N=20$ in which the ground-state (g.s.) configuration corresponds to $n \mathrm{p}-n \mathrm{~h}$ excitations belong to the so-called "island of inversion" (IoI) [11-14].

The evolution of the $N=20$ magic number has been widely studied experimentally from the $\mathrm{Ca}$ down to the $\mathrm{Na}$ nuclei 
using various techniques (see, e.g., Ref. [3] for a review and more recent references given below). From these studies it has been conjectured that magicity persists along the $N=20$ isotonic chain from ${ }^{40} \mathrm{Ca}(Z=20)$ down to ${ }^{34} \mathrm{Si}(N=14)$ [15-18] and suddenly disappears only two protons below in the deformed nuclide ${ }^{32} \mathrm{Mg}$ [19]. The recent experimental characterization of the $0_{1,2}^{+}$states in ${ }^{32} \mathrm{Mg}$ [20] and ${ }^{34} \mathrm{Si}$ [21] have provided further support for this description in which a crossing between the normal and the intruder regimes occurs between these two nuclei [22]. As discussed in the references above, the two $0^{+}$states mix in each nucleus, blurring the determination of the crossing point between normal and intruder configurations. The ground state of ${ }^{33} \mathrm{Al}(N=20)$ is, according to Refs. [23,24], outside the IoI. Therefore, ${ }^{34} \mathrm{Al}(N=21)$ offers great potential to explore this crossing for two reasons. First, two $\beta$-decaying states with spin values $4^{-}[25]$ and $1^{+}$[21] were identified, corresponding to normal $\pi\left(d_{5 / 2}\right)^{-1} \otimes v\left(f_{7 / 2}\right)$ and intruder $\pi\left(d_{5 / 2}\right)^{-1} \otimes \nu\left(d_{3 / 2}\right)^{-1}\left(f_{7 / 2}\right)^{2}$ configurations, respectively. Second, an abnormal crossover on the $S_{2 n}$ surface has been found between the $\mathrm{Al}$ and the $\mathrm{Mg}$ chains at $N=21$ [26], suggesting a structural change there.

The goal of the present Rapid Communication is to find which of these two states in ${ }^{34} \mathrm{Al}$ is the ground state and what is the energy difference between these two configurations. This will indicate how normal and intruder configurations are interleaved, and the results will be compared to the state-ofthe-art shell-model calculations that treat the nuclei using the full $s d f p$ valence space.

The ${ }^{34} \mathrm{Mg}$ ions were produced at the Isotope Separator On-Line (ISOLDE)-CERN facility through fragmentation reactions between a $1.4-\mathrm{GeV}$ pulsed proton beam delivered by the Proton Synchrotron-Booster (intervals between pulses are multiples of $1.2 \mathrm{~s}$ ) and a $\mathrm{UC}_{x}$ target. The $\mathrm{Mg}$ atoms that diffused out of the target matrix were selectively laser ionized using the resonance ionization laser ion source (RILIS) technique $[27,28]$. After being accelerated by a $40-\mathrm{kV}$ potential, the $A=34$ nuclei were selected by the ISOLDE General Purpose Separator and implanted on a movable tape, located at the center of the ISOLDE Decay Station (IDS) [29] experimental setup. The ${ }^{34} \mathrm{Mg}$ production rate of $7(1) \times 10^{2}$ ions $/ \mathrm{s}$ was estimated using $\beta-\gamma$ measurements. A $0.6 \%$ contribution of surface ionized ${ }^{34} \mathrm{Al}$ in the incoming beam was deduced when the RILIS lasers were turned off. No other contaminants could be identified based on the analysis of decay spectra. The average proton current was $1.9 \mu \mathrm{A}$, and the beam line transmission was $\sim 80 \%$. Other nuclei, such as ${ }^{34} \mathrm{Mg}$ and ${ }^{33,34} \mathrm{Al}$, were implanted directly for calibration purposes.

The beam gate was open for $200 \mathrm{~ms}$ after every proton pulse, during which ${ }^{34} \mathrm{Mg}$ nuclei were continuously implanted on the tape. The measurement of ${ }^{34} \mathrm{Mg} \beta$ decay took place at the collection point during implantation and subsequent decay. To remove the long-lived daughter activity, the tape was moved $500 \mathrm{~ms}$ after each proton pulse. In addition to this setting, two different data sets with the following conditions were collected: (i) The beam gate was reduced to $40 \mathrm{~ms}$ to obtain an accurate determination of the ${ }^{34} \mathrm{Mg}$ half-life, (ii) the beam gate was reduced to $100 \mathrm{~ms}$, and the tape was not moved in order to study the full decay chains of implanted ${ }^{33,34} \mathrm{Mg}$ and ${ }^{33,34} \mathrm{Al}$ and thus determine the number of implanted nuclei based on the neutron emission probabilities and the absolute $\gamma$-ray intensities from literature: $P_{n}\left({ }^{33} \mathrm{Mg}\right)=14(2) \%$ [30], $P_{n}\left({ }^{33} \mathrm{Al}\right)=8.5(7) \% \quad[31], \quad I_{\text {abs }}\left(1618 \mathrm{keV} ;{ }^{33} \mathrm{Mg} \rightarrow{ }^{33} \mathrm{Al}\right)=$ $16(2) \%[32]$.

$\beta$ particles were detected in a 3-cm-thick NE102 plastic scintillator made out of two joined pieces that covered a solid angle of $\sim 95 \%$ around the implantation point, whereas $\gamma$ rays were detected in five HPGe clover detectors arranged in a close geometry at $\sim 7 \mathrm{~cm}$ from the implantation point. Signals induced in the plastic scintillator were read simultaneously by two photomultiplier tubes (PMTs) placed at opposite ends. Only the events that triggered both PMTs were considered. This allowed the energy thresholds to be lowered down to around the phototube dark current in order to reach a $\beta$ efficiency close to the geometrical value. The $90(5) \% \beta$ efficiency was determined from the ratio between various $\beta$-gated and single $\gamma$ rays, in agreement with the ratio between the total number of $\beta$ decays recorded and expected in the full decay chains of ${ }^{33,34} \mathrm{Mg}$ and ${ }^{33,34} \mathrm{Al}$ when the tape was stopped. The $\gamma$-ray photopeak efficiency of the HPGe detectors was determined using the ${ }^{152}$ Eu calibration source and extrapolated using GEANT4 [33] simulations. It reached $6 \%$ at $600 \mathrm{keV}$ and $3 \%$ at $2000 \mathrm{keV}$ after the add-back procedure [34] was employed. All the signals were recorded and sampled in a triggerless mode using the 14-bit $100-\mathrm{MHz}$ Nutaq VHS-V4 data-acquisition system of the IDS [35].

The $\beta$-gated $\gamma$-ray spectrum for the $\beta$ decay of ${ }^{34} \mathrm{Mg}$, shown in Fig. 1, displays intense transitions at 364.5, 441.2, 975.0, and $1051.7 \mathrm{keV}$ in ${ }^{34} \mathrm{Al}$. The $\beta$-decay half-life of ${ }^{34} \mathrm{Mg}, T_{1 / 2}=$ 44.9 (4) $\mathrm{ms}$ was obtained from the weighted average values
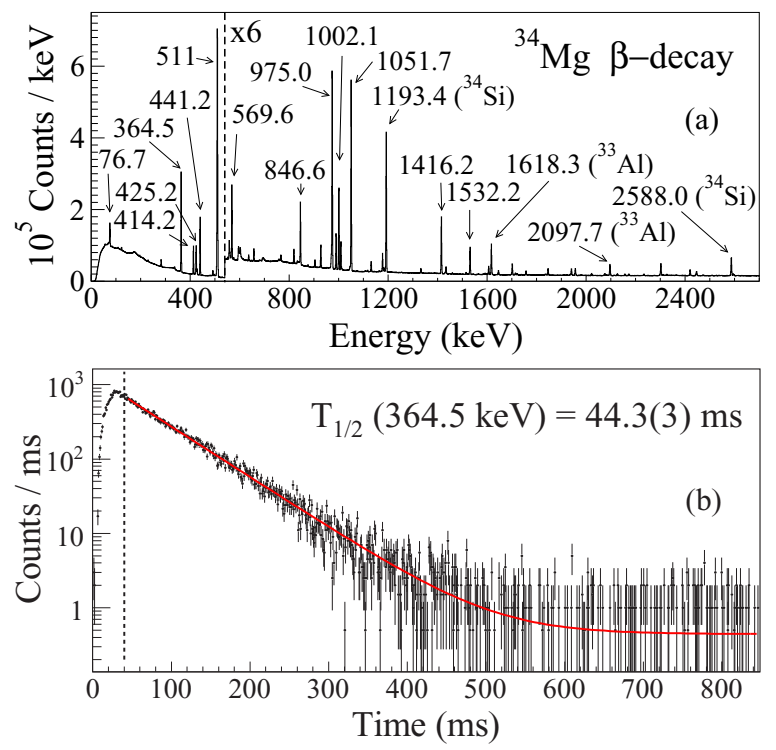

FIG. 1. (a) $\beta$-gated $\gamma$-ray spectrum of the ${ }^{34} \mathrm{Mg}$ decay recorded in the first $300 \mathrm{~ms}$ after the proton impact. The most intense transitions in ${ }^{34} \mathrm{Al},{ }^{34} \mathrm{Si}$, and ${ }^{33} \mathrm{Al}$ are labeled. (b) Time distribution, relative to the proton impact, of the $364.5-\mathrm{keV} \gamma$ ray from ${ }^{34} \mathrm{Al}$ with a corresponding decay half-life of $T_{1 / 2}=44.3(3) \mathrm{ms}$. An exponential together with a constant background were used as the fitting function, shown in red. The vertical dashed line indicates the closing of the beam gate, $40 \mathrm{~ms}$ after the proton impact. 


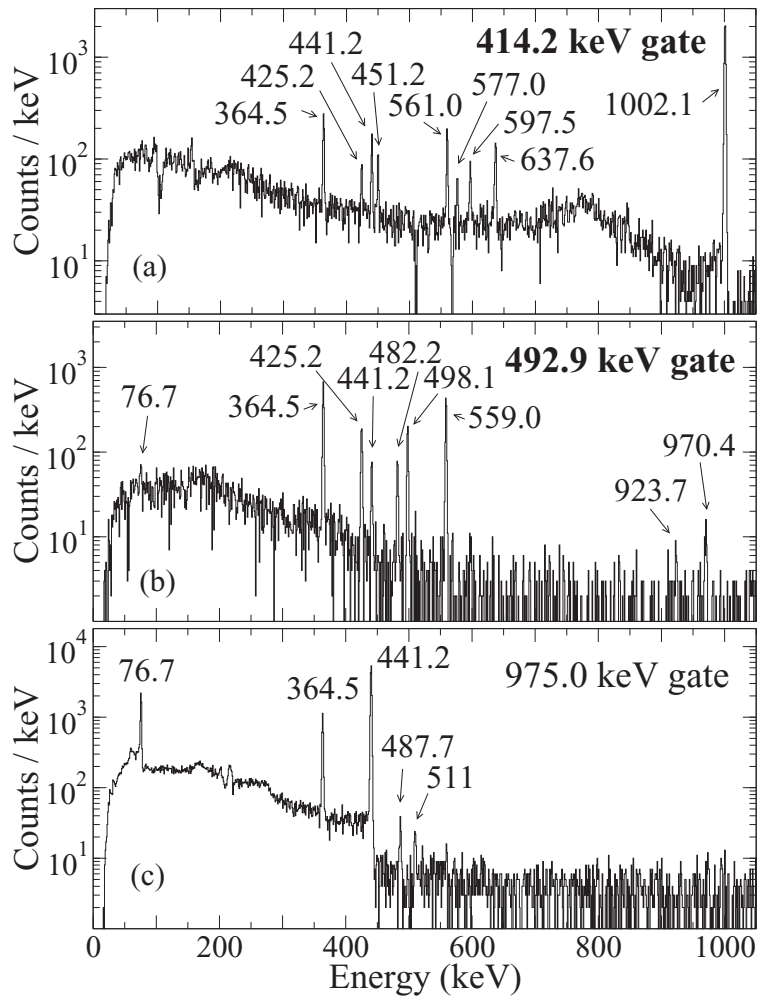

FIG. 2. Background-subtracted $\beta$-gated $\gamma$-ray spectra in coincidence with $\gamma$ rays at (a) 414.2 , (b) 492.9 , and (c) $975.0 \mathrm{keV}$ obtained from the decay of ${ }^{34} \mathrm{Mg}$. The 597.5-, 970.4- and 487.7-keV transitions feeding the $4^{-}$ground state are observed in the top, middle, and bottom spectra, respectively.

extracted by gating on these transitions (see the bottom part of Fig. 1 for a specific gate on the $364.5-\mathrm{keV}$ transition). This more precise value is twice as long as the previously measured half-life of $T_{1 / 2}=20(10) \mathrm{ms}$ [36]. It should be noted that the preliminary value of $T_{1 / 2}=63(1) \mathrm{ms}$ previously reported [37] was incorrect due to a hardware problem causing the beam gate to be fully open during the half-life measurement. New transitions belonging to the ${ }^{34} \mathrm{Mg}$ decay as well as to daughter decays through $\beta$ and $\beta$-delayed neutron emission were identified from their decay time patterns.

$\beta-\gamma$ and $\beta-\gamma-\gamma$ coincidences, some of which are shown in Fig. 2, were used to establish the level scheme of ${ }^{34} \mathrm{Al}$ shown in Fig. 3. Compared with the previous results [37], 22 new transitions are identified. In case no $\beta-\gamma-\gamma$ coincidence could be used, the placement of transitions in the level scheme was based on their relative intensity and energy summing conditions. Owing to a good energy calibration of the HPGe detectors, the determination of the excitation energy of the levels obtained from different $\gamma$-ray cascades agrees within $0.3 \mathrm{keV}$.

With a $J^{\pi}$ (g.s.) $=0^{+}$ground-state configuration, ${ }^{34} \mathrm{Mg}$ is expected to decay preferentially to $1^{+}$states in ${ }^{34} \mathrm{Al}$. The $\beta$ feeding to the ${ }^{34} \mathrm{Al} 1_{1}^{+}$of $45(8) \%$ results in a $\log f t$ value of $4.5(1)$. This absolute $\beta$ feeding together with the $\beta$-delayed neutron emission probability of $P_{n}=21(7) \%$ were derived from measurements in which the tape was not moved. An upper limit for the two-neutron emission probability $P_{2 n}<$ $0.1 \%$ has been found. This information was inferred from the deduced intensities of the $\gamma$ rays populating excited states in daughter nuclei $\left[I_{\mathrm{abs}}\left(1618 \mathrm{keV} ;{ }^{34} \mathrm{Mg} \rightarrow{ }^{33} \mathrm{Al}\right)=\right.$ $\left.13(5) \%, I_{\text {abs }}\left(364 \mathrm{keV} ;{ }^{34} \mathrm{Mg} \rightarrow{ }^{34} \mathrm{Al}\right)=11.2(15) \%\right]$ as previously mentioned. A $1_{2}^{+}$state was identified at $1462.9 \mathrm{keV}$ from its $\log f t$ value of 4.4(1), that corresponds to a GamowTeller transition. With a $\log f t$ value of $5.4(1)$, a tentative $1_{3}^{+}$ state is proposed at $2004 \mathrm{keV}$.

More than $99 \%$ of the $\beta$ decay of ${ }^{34} \mathrm{Mg}$ to bound states in ${ }^{34} \mathrm{Al}$ goes directly or indirectly (through $\gamma$ transitions from upper excited states) towards the $1_{1}^{+}$isomeric state of ${ }^{34} \mathrm{Al}$, which subsequently populates mainly the low-spin positiveparity states in ${ }^{34} \mathrm{Si}$. Feeding of the $4^{-}$state in ${ }^{34} \mathrm{Al}$ is suggested from the observation of a weak $\beta$ feeding of $<0.3(1) \%$ (the contribution of the surface ionized ${ }^{34} \mathrm{Al}$ was subtracted) to the $4^{-}$state at $4379 \mathrm{keV}$ in ${ }^{34} \mathrm{Si}$ [38]. As the direct feeding from the ground state of ${ }^{34} \mathrm{Mg}$ to the $4^{-}$state in ${ }^{34} \mathrm{Al}$ is strongly hindered by $\beta$-decay selection rules, it is probably produced through weak $\gamma$-ray cascades. The vast majority of these cascades lead to the $1_{1}^{+}$state, and their summed energies consistently amount to $1416(1) \mathrm{keV}$. However, three of them, $(414.2+451.2+$ $597.5,492.9+970.4$, and $975+487.7)$, have a summed energy of $1463(1) \mathrm{keV}$. Assuming that the $4^{-}$state lies 46.6 $\mathrm{keV}$ below the $1_{1}^{+}$state, we are able to place these transitions consistently. The 414.2, 492.9, and 975.0-keV $\gamma$-gated spectra shown in Fig. 2, prove the existence of these coincidences that probably involve transitions connecting positive- and negativeparity states as will be discussed later. Note that the ordering of the 597.5- and 451.2-keV transitions given in Fig. 3 is based on the observation of the former but not of the latter in the fragmentation study reported in Ref. [39].

Apart from the aforementioned states, the assignment of spin and parity values to other excited states is tentative. It is proposed first to identify the expected low-energy configurations of the odd-odd nuclide ${ }_{13}^{34} \mathrm{Al}_{21}$ and second to constrain spin and parity values from the many decay branches in the decay of the $1_{2}^{+}$state to the $1_{1}^{+}$or $4^{-}$states. The simplest expected configurations (1p-0h) correspond to $\pi\left(d_{5 / 2}\right)^{-1} \otimes \nu(f p)$, giving rise to negative-parity states $J^{\pi}=$ $1^{-}, \ldots, 6^{-}$or $J^{\pi}=1^{-}, \ldots, 4^{-}$when the valence neutron occupies the $f_{7 / 2}$ or the $p_{3 / 2}$ orbital, respectively.

Intruder configurations are achieved by promoting neutrons from the $d_{3 / 2}$ to the $f p$ upper shells. The promotion of one neutron corresponds to the $\pi\left(d_{5 / 2}\right)^{-1} \otimes v\left(d_{3 / 2}\right)^{-1}(f p)^{2} 2 p-$ $1 h$ configurations. They lead to positive-parity states with $J^{\pi}=1^{+}, \ldots, 4^{+}$independent of the occupancy of the paired neutrons in the $f_{7 / 2}$ or the $p_{3 / 2}$ orbital. The promotion of two and three neutrons in the upper $(f p)$ shells leads to $3 \mathrm{p}-2 \mathrm{~h}$ and $4 \mathrm{p}-3 \mathrm{~h}$ configurations with negative and positive parities, respectively. Based on this qualitative discussion and on the fact that particle-hole correlations bring extra binding energy, many states with both positive and negative parities are expected to exist in the low-energy spectrum of ${ }^{34} \mathrm{Al}$. Besides the $J^{\pi}=5^{-}$and $6^{-}$states, several others with $J=1, \ldots, 4$ can be fed directly or indirectly from the $1_{2}^{+}$state. The decay branches eventually leading to the $4^{-}$state are likely to proceed via negative-parity states. For example, the tentative $J^{\pi}=\left(2^{-}\right)$assignment of the $487.8-\mathrm{keV}$ level is based on 

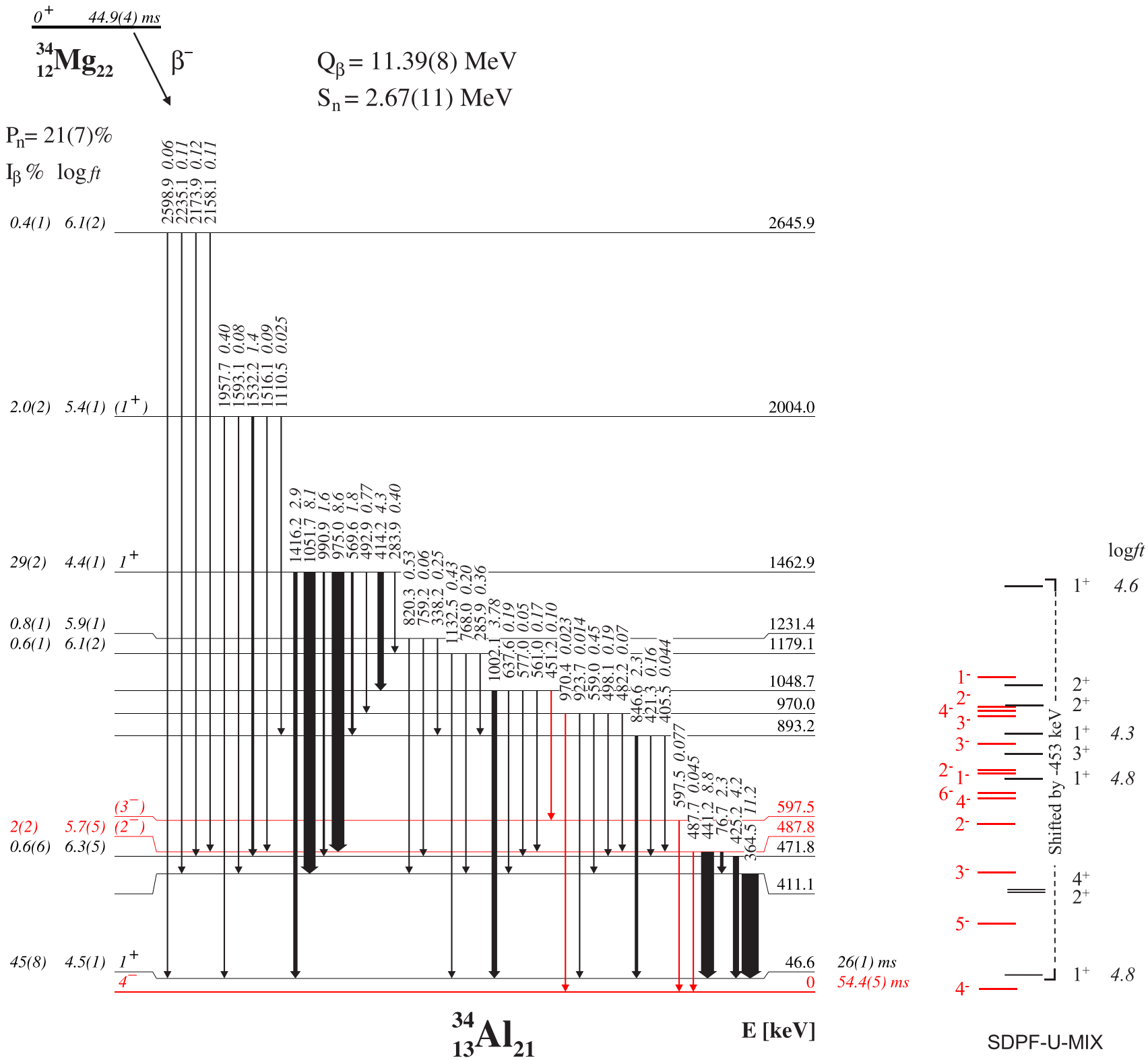

FIG. 3. Level scheme of ${ }^{34} \mathrm{Al}$ populated in the $\beta$ decay of ${ }^{34} \mathrm{Mg}$ including absolute intensities per 100 decays (left) and shell-model calculation using the SDPF-U-MIX interaction [23] (right: negative- and positive-parity states in red and black, respectively). The levels and transitions indicated in red in the experimental level scheme are linked directly to the newly identified $4^{-}$ground state and assumed to be of negative parity. Tentative spins and parities are assigned based on $\gamma$-ray branching ratios and $\log f t$ values. The half-lives of the $4^{-}$and $1^{+}$ states are taken from Ref. [21]. The calculated excitation energy of the $1_{1}^{+}$state of ${ }^{34} \mathrm{Al}$ is $\sim 500 \mathrm{keV}$. However, for display purposes all the positive-parity states were shifted down in energy so that the energy of the $1_{1}^{+}$state matches its experimental counterpart.

its direct feeding from the $1_{2}^{+}$state and its decays to both the $1_{1}^{+}$and the $4^{-}$states. The $J^{\pi}=\left(3^{-}\right)$assignment of the $597.5-\mathrm{keV}$ level results from the fact that it is not observed to be fed directly from the $1_{2}^{+}$state and it decays only to the $4^{-}$ ground state. Based on their feeding and decay patterns, two low-energy states can be assigned $J^{\pi}=2^{+}$and $J^{\pi}=1^{-}$, but their ordering cannot be determined.

In order to have a theoretical understanding of our results, we recall here the approach of Ref. [23] in which the valence space comprises the full $s d$ shell for the protons and the full $s d$ and $p f$ shells for the neutrons. The effective interaction is SDPF-U-MIX. Some of the results below were anticipated in Ref. [40]. The calculations produce for ${ }^{34} \mathrm{Al} \mathrm{a} 4^{-}$ground state dominated by the normal $1 \mathrm{p}-0 \mathrm{~h}$ configurations at $78 \%$. This is in agreement with the downward trend shown in Fig. 18 from Ref. [41] concerning the excitation energy of the lowest $4^{-}$ states in ${ }^{28,30,32} \mathrm{Al}$. The ground state of ${ }^{34} \mathrm{Al}$ contains the same amount of intruder $3 \mathrm{p}-2 \mathrm{~h}$ configurations compared to that of $2 \mathrm{p}-2 \mathrm{~h}$ in the ground state of ${ }^{33} \mathrm{Al}$. Therefore, both ground states are outside the IoI. The magnetic moment of the ${ }^{34} \mathrm{Al}$ ground state was calculated, the result being $20 \%$ smaller than the measurement reported in Ref. [25] $\mu\left(4^{-}\right)=(+) 2.156(16) \mu_{N}$. 
This could indicate that the amount of intruder mixing in the ground state of ${ }^{34} \mathrm{Al}$ is slightly higher than anticipated.

The calculation places at least 12 states of negative parity below $1 \mathrm{MeV}$. The first state dominated by the intruder $3 \mathrm{p}$ $2 \mathrm{~h}$ configurations is a $2^{-}$level at $0.58 \mathrm{MeV}$, followed by a second $4^{-}$level at $0.67 \mathrm{MeV}$ of intruder nature as well. From Fig. 3 it is difficult to establish a one-to-one correspondence between theory and experiment, beyond the agreement in the ground state spin and the presence of a low-lying triplet of states $1^{-}-3^{-}$. Notice that the separation in energy between the normal and the intruder states of negative parity is small thus we can conclude that ${ }^{34} \mathrm{Al}$ lies on the very edge of the IoI.

We have not tried to compute many states of all the possible spins, except for the $1^{+}$levels that should be fed by allowed $\beta$ decays from the $0^{+}$ground state of ${ }^{34} \mathrm{Mg}$. The calculation correctly predicts a $1^{+}$state as the lowest state dominated by $2 \mathrm{p}-1 \mathrm{~h}$ configurations at $90 \%$ and a $2^{+} 290-\mathrm{keV}$ level of $2 \mathrm{p}$ - $1 \mathrm{~h}$ nature above it, in good agreement with the experimental value of $364 \mathrm{keV}$. In addition, these two states are connected by a strong $M 1$ transition of $0.33 \mu_{N}^{2}$. The three remaining $1^{+}$levels are dominated fully by $4 \mathrm{p}-3 \mathrm{~h}$ configurations. We have computed the $\log f t$ values of the four decays with the standard quenching factor of 0.77 , and the results are shown on the right-hand side of Fig. 3. The agreement is good for the two experimentally detected decays to the first and fourth states. However the two other calculated states are absent from the experimental data or present at higher energies. This is intriguing because the density of $1^{+}$states should be much higher and because the splitting between $2 \mathrm{p}-1 \mathrm{~h}$ and $4 \mathrm{p}-3 \mathrm{~h}$ states is similar to that between $1 \mathrm{p}-0 \mathrm{~h}$ and $3 \mathrm{p}-2 \mathrm{~h}$ states.

The calculation is not able to reproduce the relative position of the negative- and positive-parity states, whose splitting is overestimated by $500 \mathrm{keV}$. In order to make the comparison easier we have displaced in Fig. 3 all the positiveparity states to line up the $1_{1}^{+}$state with its experimental counterpart. The SDPF-U-MIX places the uncorrelated $2 \mathrm{p}-1 \mathrm{~h} 1_{1}^{+}$ state $5 \mathrm{MeV}$ above the uncorrelated $1 \mathrm{p}-0 \mathrm{~h} 4_{1}^{-}$state, which reflects the $s d$-pf gap of SDPF-U-MIX at $N=20, Z=13$. Their respective total correlation energies are 8.0 and $3.5 \mathrm{MeV}$. Thus, the final splitting results from the cancellation of two large numbers 8.0 and $8.5 \mathrm{MeV}$. A precision on the order of $50 \mathrm{keV}$ as would be needed to account for the observed 46-keV energy difference between the $4^{-}$and the $1^{+}$states is out reach of the present shell-model calculation's capabilities.

In conclusion, the present Rapid Communication establishes that the $4^{-}$state of normal configuration constitutes the ground state of ${ }^{34} \mathrm{Al}$ with the intruder $1^{+}$isomer lying at an excitation energy of only $46.6 \mathrm{keV}$. This was achieved by carefully investigating through $\beta-\gamma$ and $\beta-\gamma-\gamma$ coincidences the level scheme of ${ }^{34} \mathrm{Al}$ populated in the $\beta^{-}$decay of ${ }^{34} \mathrm{Mg}$ at ISOLDE-CERN. A $\beta$-decay half-life of $T_{1 / 2}=44.9(4) \mathrm{ms}$ and a $\beta$-delayed neutron emission probability of $21(7) \%$ were derived for ${ }^{34} \mathrm{Mg}$. The small energy difference between the two $\beta$-decaying states places ${ }^{34} \mathrm{Al}$ at the crossing point between normal and intruder configurations on the edge of the $N=20$ island of inversion. State-of-the-art shell-model calculations using the SDPF-U-MIX interaction predict the interleaving of normal and intruder states in ${ }^{34} \mathrm{Al}$. The calculated excitation energy of $500 \mathrm{keV}$ for the $1^{+}$isomer is in reasonable agreement with the measurement, considering the large correlation effects in this region. These experimental results should be of great importance to benchmark future calculations around the $N=$ $20 \mathrm{IoI}$ and further away from stability.

This Rapid Communication was partially supported by a grant of the Romanian National Authority for Scientific Research and Innovation, CNCS-UEFISCDI Project No. PN-II-RU-TE-2014-4-1455, the Romanian IFA Grant CERN/ISOLDE, by FWO-Vlaanderen (Belgium), by GOA/2010/010 (BOF KU Leuven), and by the Interuniversity Attraction Poles Programme initiated by the Belgian Science Policy Office (BriX network P7/12). Support from the U.K. Science and Technology Facilities Council, the European Union Seventh Framework through ENSAR (Contract No. 262010), the MINECO (Spain) Grants No. FPA2015-64969P, No. FPA2014-57196, and No. FPA2015-65035-P, Programme "Centros de Excelencia Severo Ochoa" Program No. SEV-2012-0249, the German BMBF under Contract No. 05P15PKCIA (ISOLDE), and "Verbundprojekt 05P2015" also is acknowledged.
[1] M. G. Mayer, Phys. Rev. 75, 1969 (1949).

[2] O. Haxel, J. H. D. Jensen, and H. E. Suess, Phys. Rev. 75, 1766 (1949).

[3] O. Sorlin and M.-G. Porquet, Prog. Part. Nucl. Phys. 61, 602 (2008).

[4] K. Marinova et al., Phys. Rev. C 84, 034313 (2011).

[5] A. Gade, Eur. Phys. J. A 51, 118 (2015).

[6] T. Otsuka, R. Fugimoto, Y. Utsuno, B. A. Brown, M. Honma, and T. Mizusaki, Phys. Rev. Lett. 87, 082502 (2001).

[7] N. Smirnova et al., Phys. Lett. B 686, 109 (2010).

[8] N. A. Smirnova, K. Heyde, B. Bally, F. Nowacki, and K. Sieja, Phys. Rev. C 86, 034314 (2012).

[9] E. Caurier, G. Martínez-Pinedo, F. Nowacki, A. Poves, and A. P. Zuker, Rev. Mod. Phys. 77, 427 (2005).

[10] A. P. Zuker, J. Retamosa, A. Poves, and E. Caurier, Phys. Rev. C 52, R1741 (1995).

[11] A. Poves and J. Retamosa, Phys. Lett. B 184, 311 (1987).
[12] E. K. Warburton, J. A. Becker, and B. A. Brown, Phys. Rev. C 41, 1147 (1990).

[13] K. Heyde and J. L. Wood, J. Phys. G: Nucl. Part. Phys. 17, 135 (1991).

[14] N. Fukunishi, T. Otsuka, and T. Sebe, Phys. Lett. B 296, 279 (1992).

[15] J. Enders et al., Phys. Rev. C 65, 034318 (2002).

[16] R. W. Ibbotson et al., Phys. Rev. Lett. 80, 2081 (1998).

[17] G. Burgunder et al., Phys. Rev. Lett. 112, 042502 (2014).

[18] A. Mutschler et al., Nat. Phys. 13, 152 (2017).

[19] H. L. Crawford et al., Phys. Rev. C 93, 031303(R) (2016).

[20] K. Wimmer et al., Phys. Rev. Lett. 105, 252501 (2010).

[21] F. Rotaru et al., Phys. Rev. Lett. 109, 092503 (2012).

[22] A. O. Macchiavelli et al., Phys. Rev. C 94, 051303(R) (2016).

[23] E. Caurier, F. Nowacki, and A. Poves, Phys. Rev. C 90, 014302 (2014)

[24] H. Heylen et al., Phys. Rev. C 94, 034312 (2016). 
R. LICĂ et al.

[25] P. Himpe et al., Phys. Lett. B 658, 203 (2008).

[26] A. A. Kwiatkowski et al., Phys. Rev. C 92, 061301(R) (2015).

[27] V. Fedoseyev et al., Hyperfine Interact. 127, 409 (2000).

[28] U. Köster et al., Nucl. Instrum. Methods Phys. Res., Sect. B 266, 4229 (2008).

[29] http://isolde-ids.web.cern.ch/.

[30] J. C. Angélique et al., Spectroscopy Near the $N=20$ Shell Closure: $\beta$ - $n$ Decay Studies of ${ }^{34} \mathrm{Mg}$ and ${ }^{35} \mathrm{Al}$, AIP Conf. Proc. No. 831 (AIP, New York, 2006), p. 134.

[31] P. Reeder et al., Proceedings of the International Conference on Exotic Nuclei and Atomic Masses (ENAM-95), Arles, France, June 19-23, 1995 (INSPIRE, 1995), p. 587.

[32] V. Tripathi et al., Phys. Rev. Lett. 101, 142504 (2008).
PHYSICAL REVIEW C 95, 021301(R) (2017)

[33] S. Agostinelli et al., Nucl. Instrum. Methods Phys. Res., Sect. A 506, 250 (2003).

[34] G. Duchêne et al., Nucl. Instrum. Methods Phys. Res., Sect. A 432, 90 (1999).

[35] I. H. Lazarus et al., IEEE Trans. Nucl. Sci. 48, 567 (2001).

[36] M. Langevin et al., Nucl. Phys. A 414, 151 (1984).

[37] R. Lică et al., Properties of Low-Lying Intruder States in ${ }^{34} \mathrm{Al}$ and ${ }^{34} \mathrm{Si}$ Populated in the $\beta$ Decay of ${ }^{34} \mathrm{Mg}$ (AIP, New York, 2015).

[38] S. Nummela et al., Phys. Rev. C 63, 044316 (2001).

[39] M. Gelin, Ph.D. thesis, Université de Caen, 2007.

[40] B. Bastin et al., Phys. Rev. Lett. 99, 022503 (2007).

[41] T. A. Hinners et al., Phys. Rev. C 77, 034305 (2008). 